\title{
Surface Modification of Printed Silver Electrodes for Efficient Carrier Injection in Organic Thin-Film Transistors
}

\author{
Keisuke Aoshima, ${ }^{\mathrm{a}, \mathrm{b}}$, Shunto Arai ${ }^{\mathrm{a}}$, Katsuo Fukuhara ${ }^{\mathrm{b}}$, Toshikazu Yamada ${ }^{\mathrm{b}}$, and Tatsuo \\ Hasegawa ${ }^{\text {a,b }}$ \\ ${ }^{a}$ Department of Applied Physics, The University of Tokyo, 7-3-1 Hongo, Bunkyo-ku, Tokyo 113-0033, \\ Japan \\ ${ }^{\mathrm{b}}$ National Institute of Advanced Industrial Science and Technology (AIST), AIST Central 5, 1-1-1 \\ Higashi, Tsukuba 305-8565, Japan
}

\begin{abstract}
The modification of printed silver electrode surfaces for use as the bottom-contact electrodes of organic thin-film transistors (OTFTs) is reported. Printed silver electrodes fabricated using the surface photoreactive nanometal printing (SuPR-NaP) technique are inevitably covered with an inert surface layer of alkylamines that is originally used for encapsulation of the silver nanoparticles (AgNPs). However, it may act as a built-in protective layer against carrier injections. We demonstrate that a simple vapor exposure method is sufficient for converting the protective layer into a layer that assists carrier injection. As modifiers, we used various types of fluorinated benzenethiols that exhibit a stronger coordination with the silver surfaces than the alkylamimes. We detected the chemical conversion from alkylamine encapsulation to thiol coordination by surface enhanced Raman spectroscopy (SERS) and evaluated the improvement in the carrier injection using a transfer length method (TLM) for the OTFTs. Among the modifiers, the pentafluorobenzenethiol (PFBT) treatment significantly improves the device performance and stability of the OTFTs.
\end{abstract}

\section{Keywords}

Printed silver electrodes/ Organic thin film transistor (OTFT)/ Carrier injection/ Surface chemical modification/ Surface enhanced Raman spectroscopy (SERS)/ Transfer length method (TLM) 


\section{Introduction}

Metal-semiconductor interfaces often pose serious challenges in the manufacture of semiconductor devices. For example, carrier injection from the metals to the semiconductors are considerably affected by the formation of surface states at the inorganic semiconductor interfaces or by the blocking layers owing to the oxidized metal electrode surfaces [1]. Similar issues are also critical in printed electronics technologies, where printing techniques are used to produce lightweight, large-area, and flexible electronics devices [2,3]. A primary issue involves printed metal electrodes that are produced using "nanometal ink" that is composed of ligand encapsulated metal nanoparticles [4,5]. The use of ligand encapsulation is indispensable because metal nanoparticles can be densely suspended in the dispersion media or in the form of a fluidic ink medium only if the active (or unstable) metal surfaces are encapsulated by the insulating ligand layers that can prevent metal-metal coagulation. After printing the nanometal ink, however, these encapsulating layers not only prevent sintering between the metal nanoparticles [6,7], but also may act as protective layers against efficient carrier injections into the semiconductors.

Recently, we have successfully developed a novel printing technique called the "SuPR-NaP technique" that enables the easy, high-speed, and large-area production of ultrafine silver electrodes [8]. This process only involves the masked irradiation of vacuum ultraviolet (VUV) light on a polymer layer to photoactivate the surface and a subsequent blade coating of the alkylamine-encapsulated silver nanometal ink [4,5]. It eventually triggers the chemisorption and self-fusion reaction of the AgNPs exclusively on the photo-activated area. This technique permits the fabrication of ultrafine printed silver electrodes with a linewidth as fine as $0.8 \mu \mathrm{m}$ that strongly adheres to the substrate surfaces. However, when we use printed silver electrodes for organic thin-film transistors (OTFTs), the built-in surface alkylamine layers that inevitably cover the electrode surfaces may work as protective layers against carrier injections.

In this paper, we report a simple vapor-exposure method for chemically modifying the silver electrode surface to enable efficient carrier injection at the semiconductor-metal interface. We used benzenthiol (BT) [9], 4-fluorobenzenethiol (4-FBT) [9,10], and pentafluorobenzenethiol (PFBT) [9-13] as surface modifiers that chemically connect more strongly with the silver surfaces through the sulfur-silver contact than the alkylamines. The PFBT is known as a well-used modifier and has been used for vacuum-deposited Au electrodes [11]. In most cases, however, the electrodes are modified by being immersed in the PFBT solution. Although the immersing technique may leave contaminations or often cause damages on the electrode surfaces, the technique might have been used because it is not known to detect and confirm the completeness of surface modifications on the electrode surfaces. In contrast, we here utilized surface enhanced Raman spectroscopy (SERS) [14] to sensitively detect the chemical modifiers as chemically connected on the electrode surfaces. Note that we utilized the SERS technique to investigate the chemisorption effect of the AgNPs on the 
photo-activated surface area, and thereby established the process mechanism of the SuPR-NaP technique [8]. We demonstrate that the simple vapor-exposure method allows immediate chemical conversion from the alkylamine encapsulation to the modifiers, whose completeness on the entire electrode surface can be visualized by the SERS technique. By the modification, not only the contact resistance but also the device stability was considerably improved in the organic thin film transistors (OTFTs).

\section{Experiment}

\section{Manufacture of printed silver electrodes by the SuPR-NaP technique:}

We first prepared a patterned photoactivated surface on a substrate [8]. A thin film of amorphous perfluorinated polymer, poly [perfluoro (4-vinyloxy-1-butene)] (Cytop), was fabricated on a substrate by spin coating a diluted solution in a fluorinated solvent (CTL-809M, Asahi Glass Co., Ltd., Japan) at 2,000 rpm for $60 \mathrm{sec}$ at room temperature. The film was then dried at $180^{\circ} \mathrm{C}$ at 0.02 MPa for $60 \mathrm{~min}$. A patterned photoactivated polymer surface was produced by masked VUV irradiation (wavelength at $172 \mathrm{~nm}$ using a $\mathrm{Xe}_{2}$ eximer lamp) through a photomask. The film was set in a chamber filled with $\mathrm{N}_{2}$ gas with a residual $\mathrm{O}_{2}$ lower than $300 \mathrm{ppm}$. The typical thickness of the Cytop layer was $700 \mathrm{~nm}$. The film was irradiated by VUV light at an average dose of $6.4 \mathrm{~mW} / \mathrm{cm}^{2}$ for $80 \mathrm{sec}$.

We used silver nanometal ink [4,5] that contains AgNPs encapsulated by hexylamine, dodecylamine, $N, N$-dimethyldiaminopropane, and a few carboxylic acid; the AgNPs were densely dispersed in a 4:1 mixed solvent of octane and butanol. The typical weight fraction of the AgNPs was $50-60 \mathrm{wt} \%$ in the ink. The patterned photoactivated surface was coated by silver nanometal ink using a blade-coating technique at a sweep rate of $2 \mathrm{~mm} / \mathrm{s}$ at ambient conditions. Eventually, a thin silver layer was deposited only on the photoactivated area (Fig. 1a). In order to obtain sufficient conductivity, we dried them for a minimum of 5 hours at ambient conditions.

\section{Surface modification:}

We performed a chemical vapor modification of the printed silver electrodes to convert the built-in alkylamine layer to other electronically active layers. As modifiers, we used three kinds of benzenethiols: benzenthiol (BT) (Wako Pure Chem. Ind. Co., Ltd., 95\%), 4-fluorobenzenethiol (4-FBT) (Tokyo Chem. Ind. Co., Ltd. (TCI), 95\%), and pentafluorobenzenthiol (PFBT) (TCI, 95\%). The printed silver electrodes were exposed to the vapor of the modifier for $20 \mathrm{~min}$ at room temperature. This modification resulted in the conversion of the coordinated molecules from alkylamine to benzenethiols on the surface of the silver pattern, as schematically shown in Fig. 1a 
(center to the right).

Detection of the chemical conversion by SERS:

SERS measurements were carried out using a confocal Raman microscope (inVia RENISHAW Co. Ltd., UK). The Raman spectra were measured at an incident wavelength of $532 \mathrm{~nm}$. The incident laser power was set to a value below $1 \mathrm{~mW}$, to prevent heating. For reference, we also measured the Raman spectrum of liquid-phase BTs filled in a rectangular capillary tube with an inner size of 0.1 $\mathrm{mm} \times 1 \mathrm{~mm}$.

\section{Evaluation of the work function}

We measured the work function of the SuPR-NaP silver surface which is unmodified and modified by PFBT with photoelectron yield spectroscopy (PYS) (AC-2, RIKEN KEIKI, Japan) and Kelvin probe (KP) (FAC-01, RIKEN KEIKI, Japan). In the KP measurement, the work function of gold was referred to as $-4.83 \mathrm{eV}[10]$.

\section{Fabrication of the OTFTs:}

We manufactured bottom-gate, bottom-contact OTFTs, whose structure is indicated in Fig. 1b. The gate electrode consisting of trilayers (0.5-nm-thick $\mathrm{Cr}$, 6-nm-thick $\mathrm{Au}$, and 0.5-nm-thick $\mathrm{Al}$ ) was deposited on a quartz base plate by vacuum deposition. The top Al layer was formed to increase the adhesion to a Cytop layer that was subsequently coated. We used the Cytop layer not only as a gate-insulator but also as a layer to form a photoactivated surface. We manufactured printed source/drain electrodes on the photoactivated surface using the SuPR-NaP technique. Then, the surfaces of the source/drain electrodes were modified by BT, 4-FBT, or PFBT. We deposited pentacene (NARD Inst. Ltd., purified with sublimation thrice) to form a channel semiconductor layer by vacuum deposition. The channel length was $50-150 \mu \mathrm{m}$ and the channel width was $500 \mu \mathrm{m}$. We fabricated and evaluated 20-30 OTFTs for each modifier.

\section{Evaluation of the OTFT characteristics:}

The current-voltage characteristics of the OTFTs were measured with semiconductor parameter analyzers (E5270, Agilent Technologies). We calculated the carrier mobility $(\mu)$ in the saturation regime using the following equation:

$$
\mu=\frac{2 L}{W C}\left(\frac{\partial \sqrt{I_{\mathrm{D}}}}{\partial V_{\mathrm{G}}}\right)^{2},
$$

where $L$ is the channel length, $W$ is the channel width, $C$ is the capacitance per unit area of the dielectric, $I_{\mathrm{D}}$ is the drain current, and $V_{\mathrm{G}}$ is the gate voltage, respectively. In the linear region, a transfer length method (TLM) $[15,16]$ was employed for estimating the contact resistance $\left(R_{\mathrm{C}} \times W\right)$. 
Note that $R_{\mathrm{C}}$ is the sum of the contact resistance at the source and drain electrodes. The total resistance between the source and the drain electrodes $\left(R_{\text {total }} \times W\right)$ is indicated as:

$$
R_{\text {total }} \times W=\frac{L}{\mu C\left(V_{\mathrm{G}}-V_{\mathrm{T}}\right)}+R_{\mathrm{C}} \times W,
$$

where $V_{\mathrm{T}}$ is the threshold voltage. When we plot $R_{\text {total }} \times W$ as a function of $L$, the y-intercept of the line gives the contact resistance, $R_{\mathrm{C}} \times W$ and the slope of the line indicates the channel resistance. These values were evaluated using least square approximation.

Observation of the morphology of pentacene using AFM:

The surface morphology of the pentacene thin films was characterized using an atomic force microscope (AFM) (Dimension ${ }^{\mathrm{TM}} 3100$, diVMG, USA). We captured the tapping mode AFM images at a scan rate of $1 \mathrm{~Hz}$.

\section{Results and Discussions}

\subsection{Detection of the chemical modification}

In Fig. 2, we present the SERS spectra measured on the printed silver surfaces before and after the modification. The spectrum owing to the alkylamines was completely converted into the spectra owing to the BTs by the modification. All the peak assignments are shown in Table 1. When we compare the spectra owing to the PFBT on the electrode (Fig. 2d) and in liquid phase (Fig. 2e), we found that the peak at $2599 \mathrm{~cm}^{-1}$ assigned to the S-H stretching [18] disappears for the PFBT. The result clearly indicates that the S-H bond in the PFBT was converted into an S-Ag coordination. We also observed from Figs. $2 \mathrm{~b}$ and $2 \mathrm{c}$ that the peaks owing to S-H bond disappear in the 4-FBT and the BT on the electrode.

We used the Raman imaging technique to measure the distribution of the chemical modification with the PFBT on the electrode surface, the results of which are shown in Fig. 3. We investigated the images of the integrated Raman intensity for two different wavenumber ranges: $840-860 \mathrm{~cm}^{-1}$ (that includes a peak owing to the C-S stretching vibration in the PFBT $[19,20])$ and $2770-3025 \mathrm{~cm}^{-1}$ (that includes peaks owing to the $\mathrm{C}-\mathrm{H}$ stretching vibrations in the alkylamine $[21,22]$ ). Before the modification, SERS signals owing to the alkylamines were clearly detected on the electrode (Fig. 3b), while no signal owing to PFBT was detected (Fig. 3c). After the modification, SERS signals owing to the alkylamines are almost missing (Fig. 3d), while strong SERS signals because of the PFBT are detected on the entire electrode surface (Fig. 3e). This result clearly indicates that the built-in protective layer was chemically converted on the electrode surface. The alkylamines thus detached from the silver via the modification may temporarily stay on the surface, but should gradually volatilize as vapor. Note that the whole surface of the electrodes was completely modified via the exposure to PFBT 
vapor for only $20 \mathrm{~min}$.

\subsection{Evaluation of the contact resistivity}

In Fig. 4, we present a TLM plot for three OTFTs composed of unmodified printed silver electrodes and pentacene channel layers with different grain sizes. Note that each one of the devices, denoted as $\mathrm{A}, \mathrm{B}$, and $\mathrm{C}$ were manufactured individually. We found that the estimated contact resistances of these devices are almost same at approximately $4.3 \times 10^{7}-6.8 \times 10^{7} \Omega \mathrm{cm}$, despite significant differences in the channel resistances between $6.9 \times 10^{7}-4.0 \times 10^{8} \Omega \mathrm{cm}$ (when the channel length is $150 \mu \mathrm{m}$ ). The differences in the channel resistances are clearly associated with the differences in the morphology of the pentacene layer [26,27]. The AFM images of the channel surfaces for all the devices are shown in Fig. 4. The typical grain sizes were $500 \mathrm{~nm}$ in sample A, $1000 \mathrm{~nm}$ in sample B, and $1500 \mathrm{~nm}$ in sample C. The same tendency was also observed with the devices using surface modified printed silver electrodes (see Fig. S1 a-c). In addition, we also investigated the effect of surface modification on the pentacene grain sizes at around the electrode edges, the result of which is presented in Fig. S2. Discontinuity of the grain sizes was seen on the electrode edges for both modified and unmodified electrodes, whereas there were no obvious differences in morphology between the modified and unmodified electrode surfaces. The results indicate that the changes of contact resistance should be roughly independent of the film morphology.

In Fig. 5a, the typical transfer characteristics of devices with and without the PFBT modification of the printed silver electrodes are displayed. The carrier mobility of the OTFT improved from $2.6 \times$ $10^{-2} \mathrm{~cm}^{2} \mathrm{~V}^{-1} \mathrm{~s}^{-1}$ to $1.1 \times 10^{-1} \mathrm{~cm}^{2} \mathrm{~V}^{-1} \mathrm{~s}^{-1}$, and the hysteresis in the transfer curve was considerably suppressed. We also evaluated the contact resistance by a TLM plot, as shown in Fig. 5b. By the PFBT modification, the contact resistance improved from $2.0 \times 10^{7} \Omega \mathrm{cm}$ to $4.1 \times 10^{6} \Omega \mathrm{cm}$ (filled plots). TLM plots using device characteristics measured seven months after manufacture are depicted by open triangles and circles in Fig. 4b. It is clear that the total resistance of the OTFTs considerably increases with time, without the modification. In sharp contrast, devices using PFBT-modified printed electrodes maintain the device characteristics approximately. We also found that the PFBT modification is maintained for seven months, from the SERS spectrum shown in Fig. S3. These results indicate that the PFBT modification for the printed silver electrodes is beneficial not only for eliminating the contact resistance but also for improving the stability of the OTFT characteristics.

In Fig. 5c, we show the gate voltage dependency of the contact resistance for OTFTs with electrodes modified by different modifiers. The contact resistances of the OTFTs were obviously different, depending upon the type of modifier. A modification with BT affords a considerably larger 
contact resistance compared to an unmodified device. It has been discussed that the differences in the contact resistance by modifications using different benzenethiols should originate from the magnitude and direction of the electric dipole moment of each modifier formed on the electrode surface $[10,17]$. The dipole moments are mainly determined by the number of fluorine atoms that are the main source for drawing electrons in the molecules. The PFBT should have the strongest downward dipole moment $[10,17]$ that allows the electrode Fermi level to be lowered, reducing the Schottky barrier and improving the carrier injection at the electrode-semiconductor interface. Actually, the work function of the SuPR-NaP electrode was evaluated by PYS as $-4.43 \mathrm{eV}$ before the modification and as $-5.58 \mathrm{eV}$ after the modification (Fig. S4), respectively. Also, the values of work function measured by $\mathrm{KP}$ were $-4.63 \mathrm{eV}$ before the modification and $-5.95 \mathrm{eV}$ after the modification. These results obviously indicate that the work function of the SuPR-NaP electrode is increased by the vapor modification with PFBT. As the ionization energy of pentacene is $-5.0 \mathrm{eV}$ [28], the SuPR-NaP electrodes without any modification had higher Schottky barrier than the normal silver electrodes, whose work function is $-4.7 \mathrm{eV} \sim-4.9 \mathrm{eV}$, and the Schottky barrier should be greatly reduced by the vapor PFBT modification.

\section{Conclusion}

We investigated a method for chemically converting the built-in protective layer on printed silver electrodes produced by the SuPR-NaP technique. We found that a simple vapor exposure of thiol-based modifiers results in an almost complete modification of the printed silver electrode surface, as confirmed by SERS measurements. Among a variety of modifiers, we found that the PFBT is beneficial for considerably improving the carrier injections and the device stabilities of the OTFTs, where the Schottky barriers are suppressed by the formation of electric dipole layers at the electrode-semiconductor interfaces. The use of this simple and easy surface modification for the printed silver electrodes in the production of lightweight, large-area, and flexible electronic products would be immensely beneficial.

\section{Acknowledgement}

This work was partly supported by the Japan Science and Technology Agency (JST) through the Strategic Promotion of the Innovative Research and Development Program (S-Innovation). 


\section{References}

[1] E. H. Rhoderick and R. H. Williams, Metal-Semiconductor Contacts $2^{\text {nd }}$ ed., Oxford University Press, New York, USA (1988).

[2] A. Kamyshny and S. Magdassi, Small 10 (2014) 3515-3535.

[3] B. Kang, W. H. Lee and K. Cho, ACS Appl. Mater. Interfaces 5 (2013) 2302-2315.

[4] M. Itoh, T. Kakuta, M. Nagaok, Y. Koyama, M. Sakamoto, S. Kawasaki, N. Umeda, M. Kurihara, J. Nanosci. Nanotechnol. 9 (2009) 1-6.

[5] M. Chen, Y. G. Feng, X. Wang, T. C. Li, J. Y. Zhang and D. J. Qian, Langmuir 23 (2007) 5296-5304.

[6] S. Wünscher, R. Abbel, J. Perelaer and U. S. Schubert, J. Mater. Chem. C 2 (2014)1023210261.

[7] M. J. Coutts, M. B. Cortie, M. J. Ford and A. M. McDonagh, J. Phys. Chem. C 113 (2009) $1325-1328$.

[8] T. Yamada, K. Fukuhara, K. Matsuoka, H. Minemawari, J. Tsutsumi, N. Fukuda, K. Aoshima, S. Arai, Y. Makita, H. Kubo, T. Togashi, M. Kurihara and T. Hasegawa, Nat. Commun. 7 (2016) 11402.

[9] J. P. Hong, A. Y. Park, S. Lee, J. Kang, N. Shin and D. Y. Yoon, Appl. Phys. Lett. 92 (2008) 2006-2009.

[10] Y. Kuzumoto and M. Kitamura, Appl. Phys. Express 7 (2014) 35701.

[11] X. Wang, M. Qin, M. Yuan, X. Gu, L. Qiu, G. Zhang, J. Hu, H. Lu and G. Lv, J. Disp. Tech. 11 (2015) 450-455.

[12] M. H. Choi and J. Jang, Curr. Appl. Phys. 12 (2012) e6-e7.

[13] S. H. Kim, I. Kang, Y. G. Kim, H. R. Hwang, Y. H. Kim, S. K. Kwon and J. Jang, J. Mater. Chem. C. 1 (2013) 2408-2311.

[14] K. Kneipp, Y. Wang, H. Kneipp, L. T. Perelman, I. Itzkan, R. R. Dasari and M. S. Feld,. Phys. Rev. Lett., 78 (1997) 1667-1670.

[15] Y. Xu, R. Gwoziecki, I. Chartier, R. Coppard, F. Balestra and G. Ghibaudo, Appl. Phys. Lett. 97 (2010) 95-98.

[16] D. Gupta, M. Katiyar and D. Gupta, Org. Electron. 10 (2009) 775-784.

[17] S. Lal, N. K. Grady, J. Kundu, C. S. Levin, J. B. Lassiter and N. J. Halas, Chem. Soc. Rev. 37 (2008) 898-911.

[18] R. L. Garrell, C. Szafranski, and W. Tanner, Technology 1336 (1990) 264-271.

[19] M. C. Schalnat and J. E. Pemberton, Langmuir 26 (2010) 11862-11869.

[20] J. H. S. Green, D. J. Harrison and C. P. Stockley, Spectrochim. Acta A 33 (1977) 423-427. 
[21] N. A. Atamas, A. M. Yaremko, T. Seeger, A. Leipertz, A. Bienko, Z. Latajka,, H. Ratajczak, A. J. Barnes, J. Mol. Struct. 708 (2004) 189-195.

[22] J. C. Valmalette, Z. Tan, H. Abe and S. Ohara, Sci. Rep. 4 (2014) 5238.

[23] N. Leopold and B. Lendl, J. Phys. Chem. B 107 (2003) 5723-5727.

[24] J. Zhang, X. Li, X. Sun and Y. Li, J. Phys. Chem. B, 109 (2005) 12544-12548.

[25] R. D. Shelton, J. W. Haas and E. A. Wachter, Appl. Spectroscopy 48 (1994) 1007-1010.

[26] J. Kim, S. C. Lee, H. S. Lee, C. W. Kim and W. H. Lee, Phys. Mater. Appl. 29 (2016) 7-12.

[27] D. J. Gundlach, Y. Y. Lin, T. N. Jackson, S. F. Nelson and D. G. Schlom, IEEE Electr. Device L. 18 (1997) 87-89.

[28] N. Koch, I. Salzmann, R. L. Johnson, J. Pflaum, R. Friedlein, J. P. Rabe, Org. Electron. 7 (2006) 537-545.

\section{Figure}

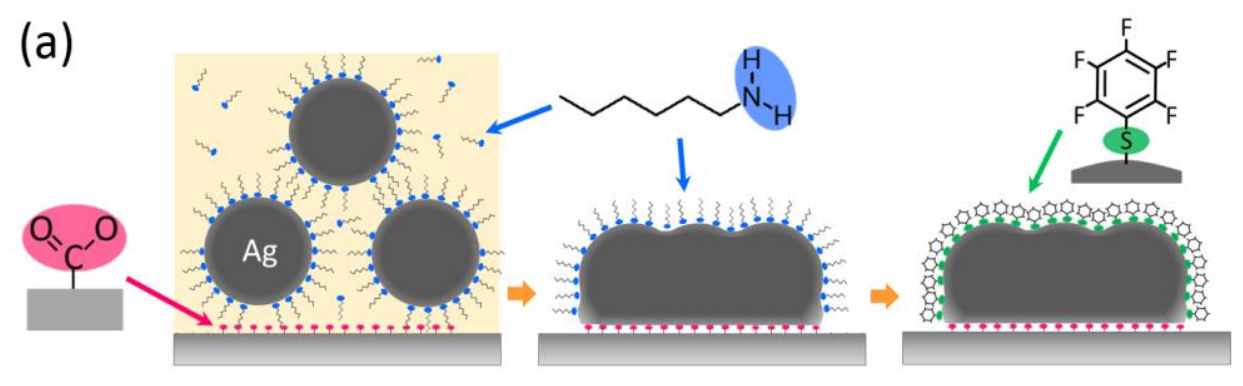

(b)

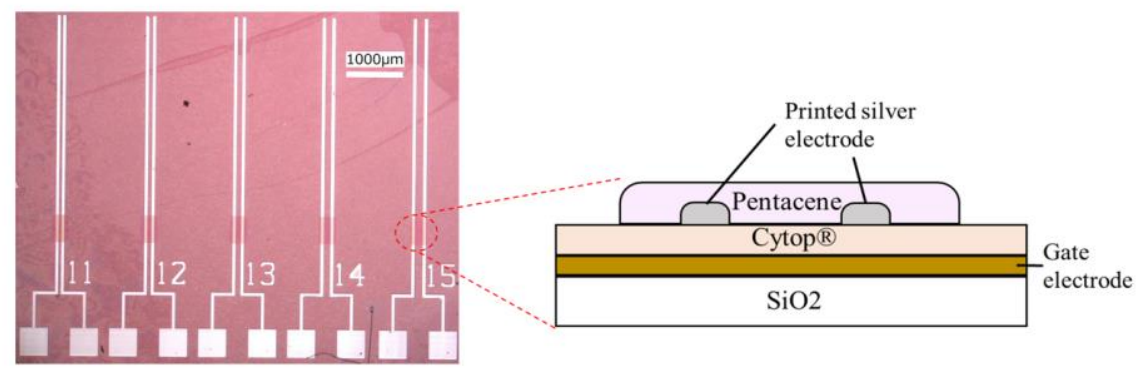

Fig. 1. (a) Schematic of the chemical conversion process. Silver nanometal ink is coated on the photoactivated surface with a carboxylate group (left). The AgNPs encapsulated by the alkylamines in the ink are exclusively chemisorbed on the photoactivated surface to form a self-fused solid silver layer (center). Vapor exposure of the modifiers enables the conversion of the built-in alkylamine layer into the modifier layer (right). (b) Photomicrograph (left) and schematic (right) of the fabricated bottom-gate, bottom-contact, pentacene OTFTs. 


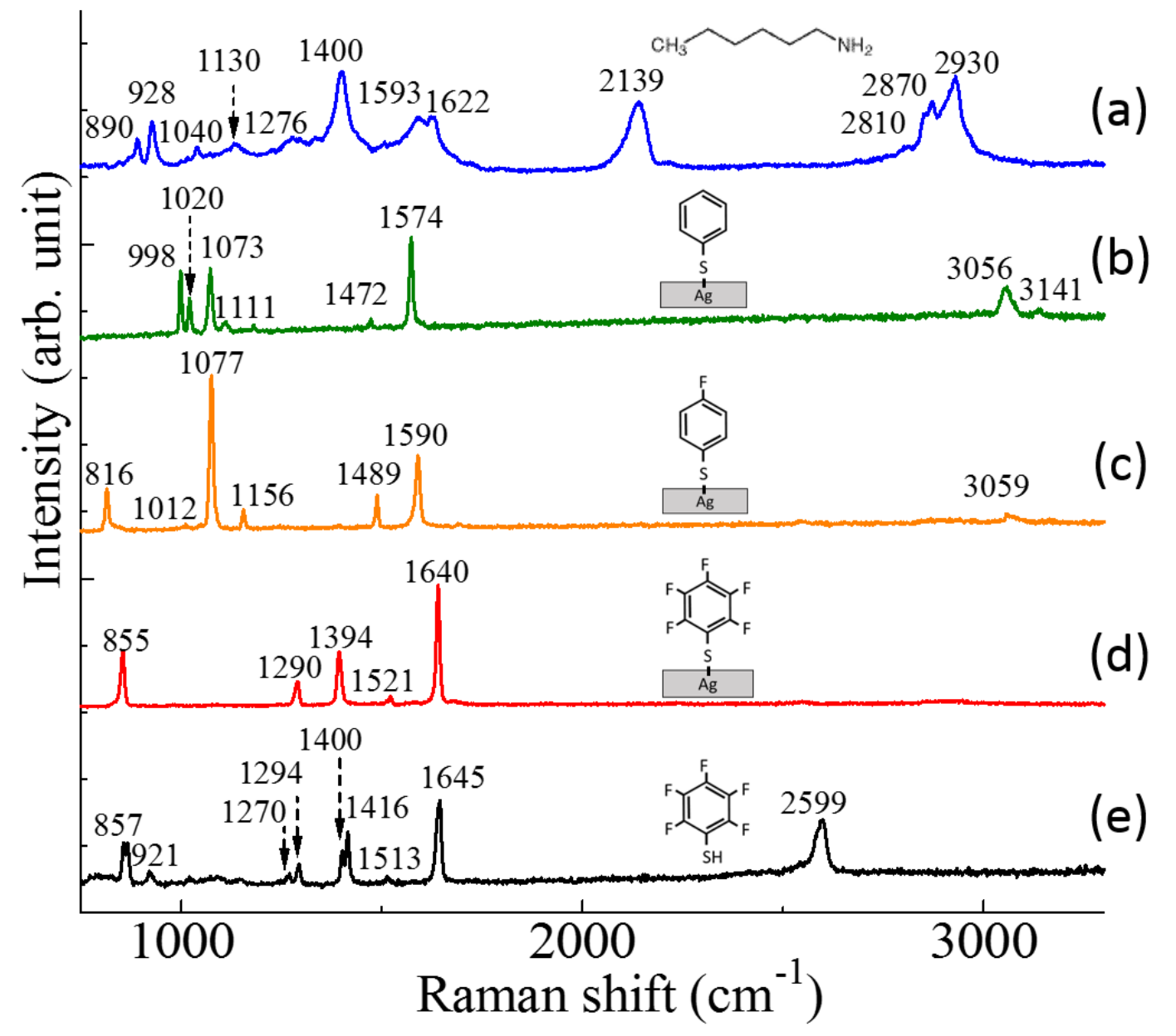

Fig. 2. Raman spectra of the (a) unmodified printed electrode surface and the surfaces modified by (b) BT, (c) 4-FBT, (d) PFBT, and of the (e) liquid-phase PFBT. Each Raman spectrum sensitively detects the chemical species coordinated on the electrode surface. 


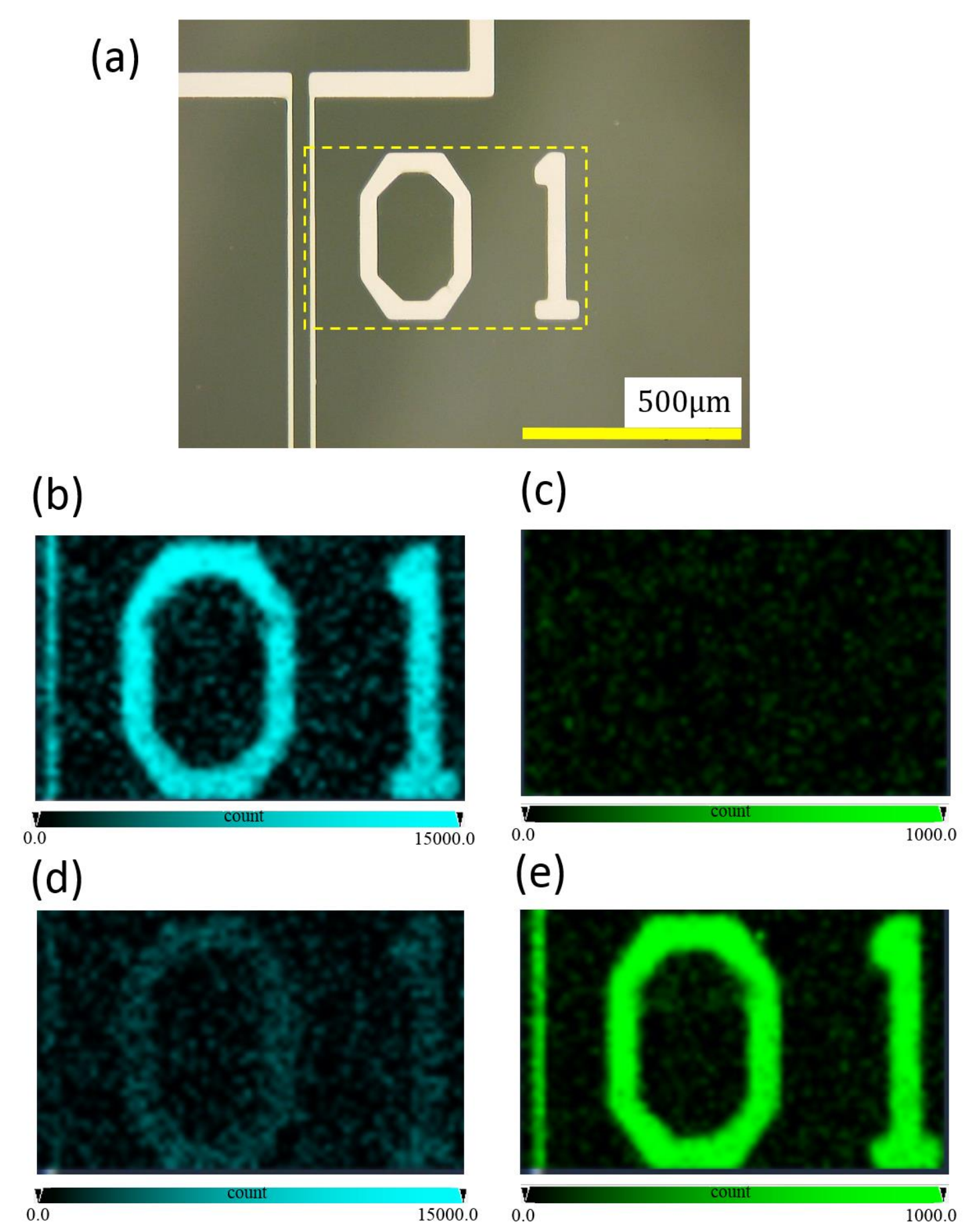

Fig. 3. (a) Photomicrograph of the printed silver electrodes. The area enclosed by the dashed line indicates the integrated Raman intensity 2D-image mapped area shown in (b)-(e). (b) and (c) display the Raman images before the modification, and (d) and (e) show the images after the modification. The wavenumber range is between $840-860 \mathrm{~cm}^{-1}$ in (b) and (d), and between $2770-3025 \mathrm{~cm}^{-1}$ in (c) and (e). The former is to detect the alkylamines and the latter is to detect the PFBT. 


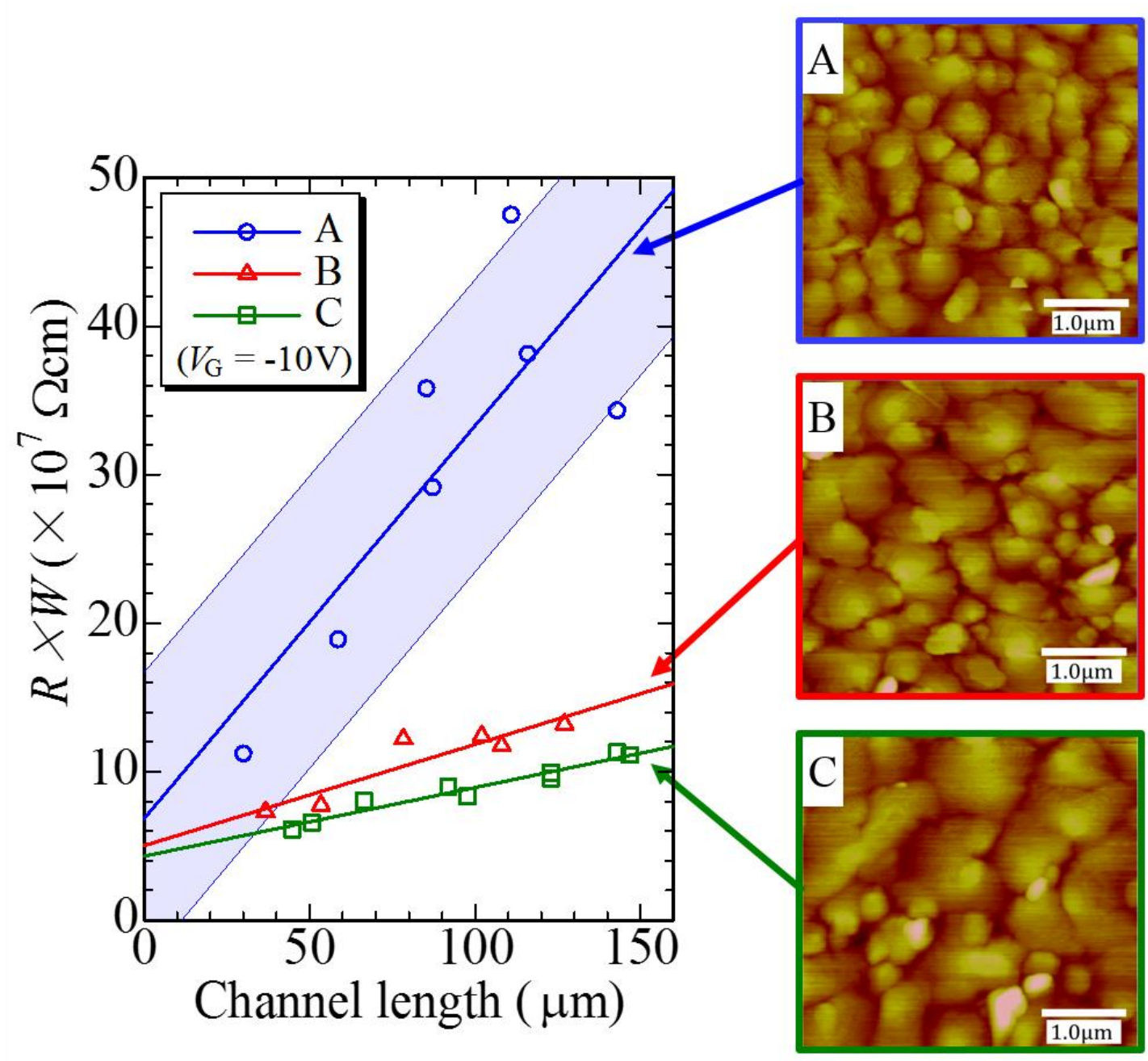

Fig. 4. Left: TLM plots of the bottom-contact, bottom gate, pentacene OTFTs with unmodified printed silver electrodes. The calculated channel resistance at $L=150 \mu \mathrm{m}$ was $4.0 \times 10^{8} \Omega \mathrm{cm}$ in sample A, $1.0 \times 10^{8} \Omega \mathrm{cm}$ in sample $B$, and $6.9 \times 10^{7} \Omega \mathrm{cm}$ in sample C. The contact resistance was $6.8 \times 10^{7} \Omega \mathrm{cm}$ in sample A, $5.0 \times 10^{7} \Omega \mathrm{cm}$ in sample B, and $4.3 \times 10^{7} \Omega \mathrm{cm}$ in sample C. Right: Surface morphology of the pentacene thin films. 


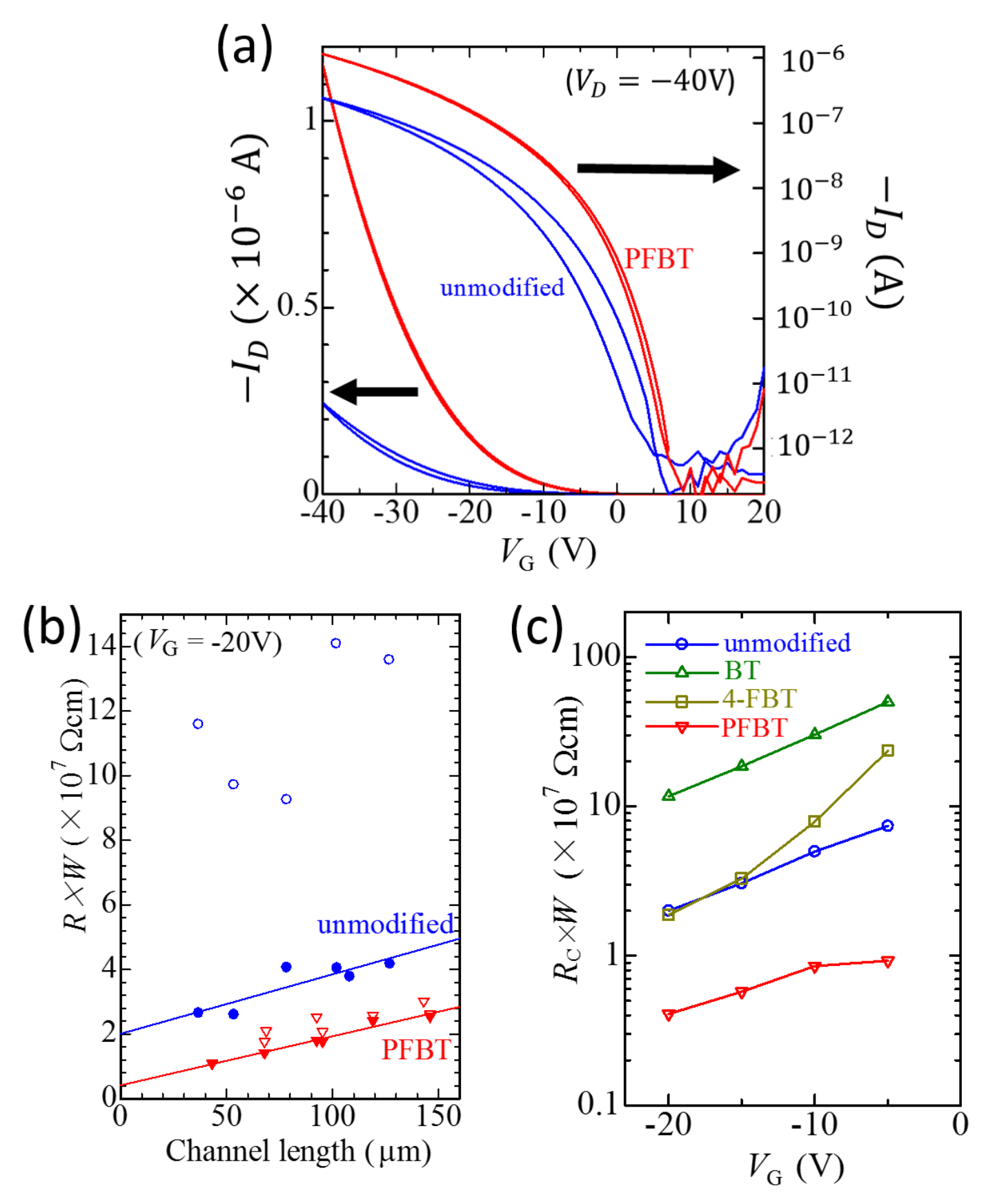

Fig. 5. (a) Transfer characteristics of the pentacene OTFTs with the printed silver electrodes with (red lines) and without (blue lines) the PFBT modification. (b) TLM plots for the OTFT characteristics with (red lines) and without (blue lines) the PFBT modification. The filled symbols indicate data immediately after the device fabrication and the open symbols represents data measured seven months after the device fabrication. (c) Gate voltage dependency of the contact resistance for OTFTs with electrodes modified by different modifiers: BT (green), 4-FBT (yellow), and PFBT (red). The results of the unmodified electrode (blue) are also plotted. 
Table

\begin{tabular}{|c|c|c|c|c|c|}
\hline \multicolumn{2}{|c|}{ Before modified } & \multicolumn{2}{|c|}{ BT-modified } & \multicolumn{2}{|c|}{ 4-FBT-modified } \\
\hline $\begin{array}{l}\text { Raman shift } \\
\qquad\left(\mathrm{cm}^{-1}\right)\end{array}$ & assignment & $\begin{array}{c}\text { Raman shift } \\
\quad\left(\mathrm{cm}^{-1}\right)\end{array}$ & assignment & $\begin{array}{l}\text { Raman shift } \\
\qquad\left(\mathrm{cm}^{-1}\right)\end{array}$ & assignment \\
\hline 890 & $v(C-C)$ & 998 & $\delta(C-C-C)_{i p}$ & 816 & $v\left(\mathrm{C}_{6} \mathrm{H}_{4} \mathrm{~F}-\mathrm{S}\right)$ \\
\hline 928 & $\delta\left(-\mathrm{CH}_{2}-\right)_{\mathrm{oop}}$ & 1020 & $\delta(\mathrm{C}-\mathrm{H})_{\text {ip }}$ & 1012 & $\delta(\mathrm{C}-\mathrm{H})_{\mathrm{ip}}$ \\
\hline 1040 & $\delta\left(-\mathrm{CH}_{2}-\right)_{\mathrm{ip}}$ & 1073 & $\begin{array}{l}v(C-C)_{\text {ring }} \\
+v(C-S)\end{array}$ & 1077 & $\begin{array}{l}v(C-C)_{\text {ring }} \\
+v(C-S)\end{array}$ \\
\hline 1130 & $\delta\left(-\mathrm{NH}_{2}\right)$ & 1111 & n. a. & 1156 & n. a. \\
\hline 1276 & $v(C-C)$ & 1180 & $\delta(\mathrm{C}-\mathrm{H})_{\mathrm{ip}}$ & & \\
\hline 1400 & $v\left(\mathrm{COO}^{-}\right)$ & 1472 & $v(C=C)$ & 1489 & $v(C=C)$ \\
\hline 1593 & $\delta\left(-\mathrm{NH}_{2}\right)$ & 1574 & $v(C=C)$ & 1590 & $v(C=C)$ \\
\hline 1622 & $v(C-N)$ & & & & \\
\hline 2139 & $v(C \equiv N)$ & & & & \\
\hline 2810 & $v\left(-\mathrm{CH}_{3}\right)$ & & & & \\
\hline 2870 & $v\left(-\mathrm{CH}_{3}\right)$ & 3056 & $v(\mathrm{C}-\mathrm{H})$ & 3059 & $v(\mathrm{C}-\mathrm{H})$ \\
\hline 2930 & $v\left(-\mathrm{CH}_{2}-\right)$ & 3141 & $v(\mathrm{C}-\mathrm{H})$ & & \\
\hline
\end{tabular}

\begin{tabular}{cccc}
\hline \multicolumn{2}{c}{ PFBT-modified } & \multicolumn{2}{c}{ PFBT (liquid) } \\
\hline $\begin{array}{c}\text { Raman shift } \\
\left(\mathrm{cm}^{-1}\right)\end{array}$ & assignment & $\begin{array}{c}\text { Raman shift } \\
\left(\mathrm{cm}^{-1}\right)\end{array}$ & assignment \\
\hline 855 & $v\left(\mathrm{C}_{6} \mathrm{~F}_{5}-\mathrm{S}\right)$ & 857 & $v\left(\mathrm{C}_{6} \mathrm{~F}_{5}-\mathrm{S}\right)$ \\
& & 921 & $\delta(\mathrm{C}-\mathrm{S}-\mathrm{H})$ \\
& & 1270 & $v(\mathrm{C}-\mathrm{F})$ \\
1290 & $v(\mathrm{C}-\mathrm{F})$ & 1294 & $v(\mathrm{C}-\mathrm{F})$ \\
1394 & $v(\mathrm{C}-\mathrm{F})$ & 1400 & $v(\mathrm{C}-\mathrm{F})$ \\
& & 1416 & $v(\mathrm{C}-\mathrm{F})$ \\
1521 & $v(\mathrm{C}=\mathrm{C})$ & 1513 & $v(\mathrm{C}=\mathrm{C})$ \\
1640 & $v(\mathrm{C}=\mathrm{C})$ & 1645 & $v(\mathrm{C}=\mathrm{C})$ \\
& & 2599 & $v(\mathrm{~S}-\mathrm{H})$
\end{tabular}

Table 1. Assignment of the Raman peaks in Fig. 2. We assigned the Raman peaks following the references [14-22]. A peak at $1400 \mathrm{~cm}^{-1}$ before modification was assigned as the stretching vibration of the carboxylic group [14] that may be derived from the ligands included in the nanometal ink or the photoactivated surface. 


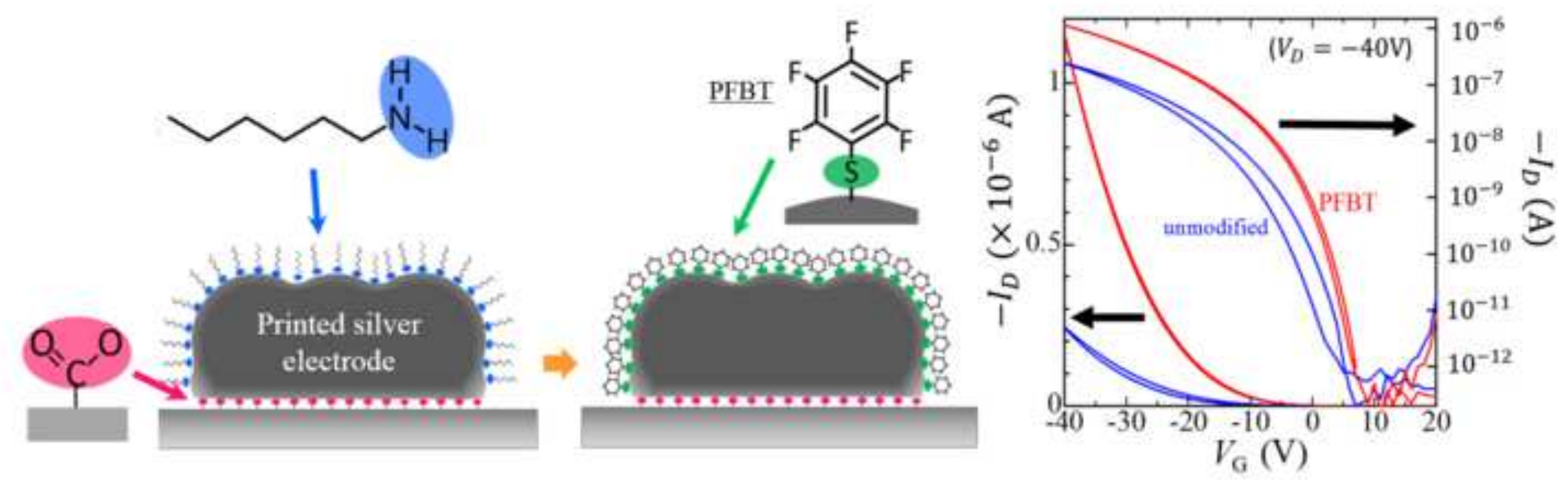

